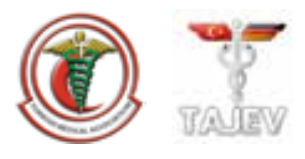

\title{
Robotic surgery in gynecology
}

\author{
Ibrahim Alkatout, Liselotte Mettler, Nicolai Maass, Johannes Ackermann \\ Department of Obstetrics and Gynecology, University Hospitals Schleswig-Holstein, Campus Kiel, Kiel, Germany
}

\begin{abstract}
Robotic surgery is the most dynamic development in the sector of minimally invasive operations currently. It should not be viewed as an alternative to laparoscopy, but as the next step in a process of technological evolution. The advancement of robotic surgery, in terms of the introduction of the Da Vinci Xi, permits the variable use of optical devices in all four trocars. Due to the new geometry of the "patient cart," an operation can be performed in all spatial directions without re-docking. Longer instruments and the markedly narrower mechanical elements of the "patient cart" provide greater flexibility as well as access similar to those of traditional laparoscopy.

Currently, robotic surgery is used for a variety of indications in the treatment of benign gynecological diseases as well as malignant ones. Interdisciplinary cooperation and cooperation over large geographical distances have been rendered possible by telemedicine, and will ensure comprehensive patient care in the future by highly specialized surgery teams. In addition, the second operation console and the operation simulator constitute a new dimension in advanced surgical training. The disadvantages of robotic surgery remain the high costs of acquisition and maintenance as well as the laborious training of medical personnel before they are confident with using the technology.
\end{abstract}

(J Turk Ger Gynecol Assoc 2016; 17: 224-32)

Keywords: Robotic surgery, laparoscopy, gynecological oncology, surgical training, cost efficiency

Received: 30 June, 2016 Accepted: 27 September, 2016

\section{Robotic surgery in gynecology}

Robotic surgery is a dynamic development for minimally invasive procedures. The specialty of gynecology consistently offers new opportunities for innovative surgical techniques and the advancement of existing therapy approaches (Figure 1).

Ever since the American FDA granted approval of the Da Vinci operation robot for gynecological operations in 2005, about three million robotic operations have been performed worldwide. 3500 Da Vinci systems are currently in use: 586 of these in Europe and 77 in Germany (4 ${ }^{\text {th }}$ quarter of 2015). According to the figures of Intuitive Surgical, about 600,000 interventions were performed on a worldwide basis in the year 2014, of which 50\% were performed in gynecology, approximately $30 \%$ in urology, and about $20 \%$ in general and chest surgeries. In 2011, the proportion of robotic hysterectomies performed for benign indications in the USA was as high as $27 \%$ (1).

Currently, we have experience in robotic surgery for the majority of gynecological operations and fields of application. The known advantages of minimally invasive surgery, such as less blood loss, shorter durations of hospital stay, and lower patient morbidity compared to open procedures, have been observed here as well. Better exposure of the operating field by $3 \mathrm{D}$ technology and the extension of surgical instruments to 7 degrees of freedom permit the use of minimally invasive surgery, even for complex indications. Robot-assisted manipulation of the instruments permits tremor-free handling and reduces work fatigue for the surgeon, which is very advanta- geous for the surgeon as well as the patient in long and complex interventions. The possibility of working simultaneously on two parallel consoles shortens the learning curve, reduces complication rates, and facilitates the training of surgeons (2). The advancement of robotic surgery in terms of the Da Vinci $\mathrm{Xi}$ permits the variable use of optics in all four trocars (paraaortic lymphadenectomy, omentectomy, or interdisciplinary surgery in the upper abdomen can be performed without re-docking) and ensures markedly greater flexibility due to the optimized geometry of the so-called patient cart. Robotic surgery has been criticized for the fact that it requires the use of larger trocars compared to conventional laparoscopy, and is therefore associated with more numerous and larger cosmetic scars; this is avoided by the smaller trocars now used in robotic surgery (3). The development of the single-site systems signifies further new options for the gynecological surgeon (Figure 2). For instance, freedom of movement is now maximized by the introduction of one or more additional working trocars $(4,5)$.

The low level of postoperative pain appears to be another advantage. It is accompanied by a lesser need for analgesics and even shorter hospital stays compared to traditional laparoscopic surgery. One explanation could be the fact that the abdominal wall need not be used as a counter bearing. The absence of irritation and the advantage of tissue protection seem to display very positive effects $(6,7)$.

The disadvantages, on the other hand, are the still high costs of installation and maintenance, the longer operating times 


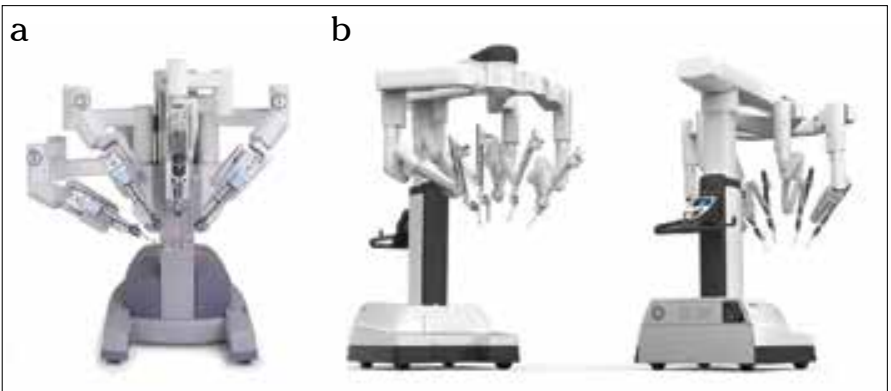

Figure 1. a, b. Da Vinci Si system with its central optical arm (12 mm diameter) and 3 working trocars, each $8 \mathrm{~mm}$ in diameter (a). The Da Vinci Xi system is markedly smaller with four identical flexible arms (8 mm each) (b). The camera and working trocars can be exchanged as desired. The simpler handling of the console and the instruments is not visible here.
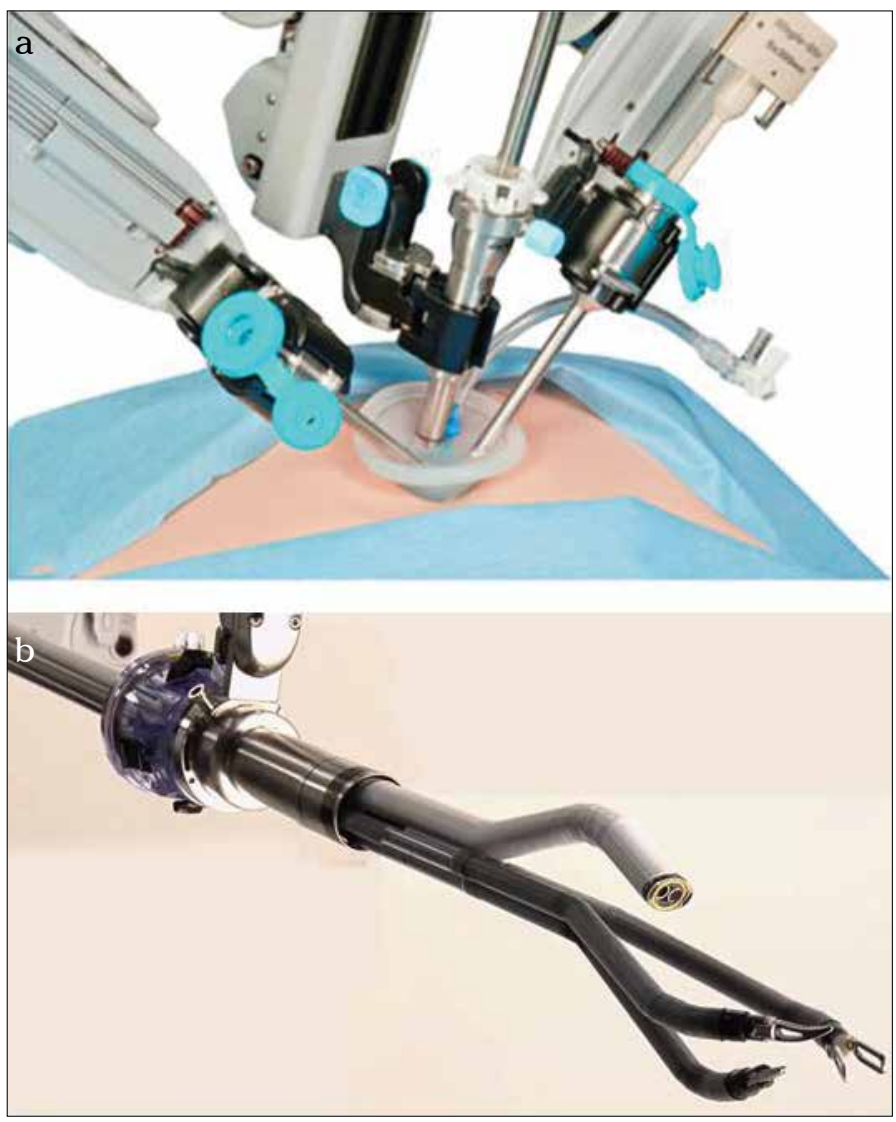

Figure 2. a, b. Single-site laparoscopy with the Da Vinci Xi system. An overview of the robot arms lying close to each other and the better distance because of the much smaller Xi system (a) Intraabdominal operations can be performed with angulated optical instruments and surgical instruments within a very small space without loss of quality (b). The single port is placed in the navel and additional working trocars can be placed at various sites in the abdominal wall to support the operation.

(at least in the beginning), and the initial learning curve that has to be traversed again (even by experienced laparoscopists) in order to work safely and gently. Doctors (anesthetists and surgeons) as well as nursing staff have to be trained for working
Table 1. Advantages of the Da Vinci robot system compared to conventional laparoscopy

\begin{tabular}{|l|}
\hline $\begin{array}{l}\text { Advantages of the Da Vinci robot system compared to } \\
\text { conventional laparoscopy }\end{array}$ \\
\hline Ergonomics \\
\hline Intuitive handling of instruments \\
\hline 3-D optics without additional equipment \\
\hline 7 degrees of freedom \\
\hline Faster learning curve \\
\hline Digital networking \\
\hline Dual console \\
\hline Integrated fluorescence visualization (Firefly) \\
\hline Less postoperative pain \\
\hline
\end{tabular}

Table 2. Disadvantages of the $\mathrm{Da}$ Vinci robot system compared to conventional laparoscopy

\begin{tabular}{|l|}
\hline $\begin{array}{l}\text { Limitations of the Da Vinci robot system compared to } \\
\text { conventional laparoscopy }\end{array}$ \\
\hline Higher costs of disposable materials and maintenance \\
\hline Absence of tactile feedback \\
\hline Additional learning curve \\
\hline Additional time for docking \\
\hline $\begin{array}{l}\text { Usually requires more numerous and larger puncture sites } \\
\text { (Si System) }\end{array}$
\end{tabular}

with the robot system $(2,5)$. The advantages and limitations of robotic laparoscopy are shown in Table 1, 2 .

\section{Application in benign gynecological diseases}

The use of robotic surgery for gynecological diseases extends to nearly all surgical therapy options. However, owing to the high cost of robotic surgery, it will probably not become a common procedure for the treatment of benign diseases. Currently, robotic surgery is focused on malignant diseases whose costs are borne more easily by the health care system. Nevertheless, it would be necessary to develop innovative operation concepts that make robotic surgery a viable option in benign disease treatment as well. (1, 8). The new technical advancements of the surgery robot may help to extend the use of minimally invasive procedures to complex fields of application that were previously accessible only to laparotomy while utilizing the advantages of the minimally invasive approach. Potential areas of use for robotic surgery in benign gynecological diseases are shown in Table 3.

Robotic hysterectomy for benign diseases is currently the alternative to conventional laparoscopy in many developed countries. The frequent use of robotic surgery has further reduced the number of abdominal hysterectomies, especially in the USA (Figure 3). Cases of complex comorbidities, such as severe adhesions, obesity, or deep infiltrating endometriosis, are well suited for robot-assisted surgery (9). Lim et al. (10) 
Table 3. Applications of Da Vinci robotic surgery for benign gynecological disease

\begin{tabular}{|l|l|}
\hline Use of robotic surgery for malignant gynecological disease \\
\hline Cervical cancer & $\begin{array}{l}\text { Radical hysterectomy } \\
\text { Total mesometrial resection (TMMR) } \\
\text { Trachelectomy }\end{array}$ \\
\hline Endometrial cancer & $\begin{array}{l}\text { Hysterectomy } \\
\text { Peritoneal mesometrial resection (PMMR) }\end{array}$ \\
\hline $\begin{array}{l}\text { Lymphadenectomy } \\
\text { and sentinel biopsy } \\
\text { (dye and fluorescence) }\end{array}$ & Pelvic lymphadenectomy \\
\hline Exenteration & Paraaortic lymphadenectomy \\
\hline Ovarian cancer & $\begin{array}{l}\text { Pnterior exenteration } \\
\text { Posterior exenteration }\end{array}$ \\
\hline Omentectomy & De-bulking advanced ovarian carcinoma \\
\hline
\end{tabular}

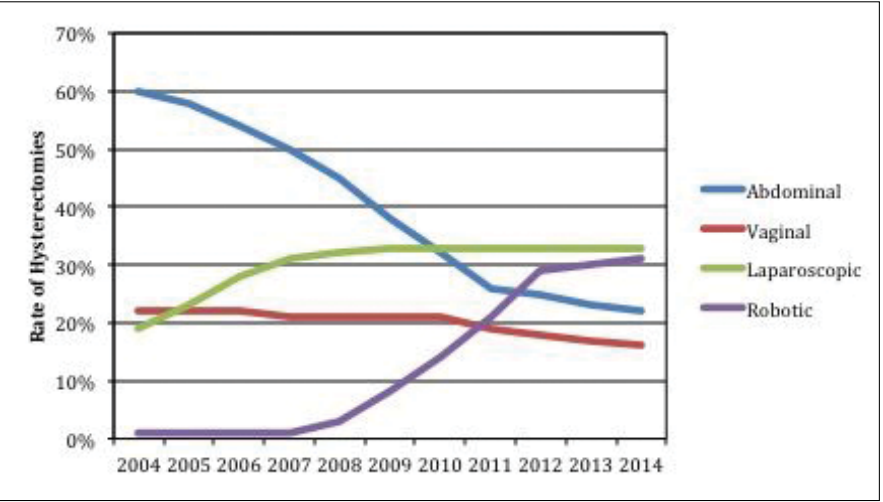

Figure 3. Trends in the use of hysterectomy methods in Western countries

published the first comprehensive multicenter study on this subject, which was only focused on experienced surgeons ( $>60$ operations), thus circumventing the distorting effect of the learning curve. The authors analyzed more than 30,000 hysterectomies with a 30-day follow-up period. Robotic hysterectomy for benign disease was compared with alternative surgical modalities. Even in complex operations, robotic hysterectomies were associated with a lower intraoperative complication rate compared to abdominal and vaginal hysterectomies, and a significantly lower postoperative complication rate compared to other surgical procedures, especially laparoscopy.

In patients with a symptomatic myomatous uterus of reproductive age, or in women who undergo the surgical procedure because they wish to have children, enucleation of the myoma is an organ-preserving therapy option (11). One of the most dreaded complications of this operation is rupture of the uterus. Due to its simplified suturing method, and the possibility of uterine reconstruction, robotic surgery yields similar results and is associated with similar rupture rates as the open procedure, especially in this setting. In a population of 107 patients, Pitter et al. (12) reported 92 births and only one case of uterine rupture. Since these patients are still young, they benefit greatly from the minimally invasive approach in terms of long-term sequela, such as adhesions or weakness of the abdominal wall. Deep infiltrating endometriosis is one of the most suitable indications for robotic surgery in benign gynecological disease treatment. Since the patients are still young and wish to have children, they require organ-preserving and tissue-protective surgery (13). Continuity is usually preserved during excision of the endometriosis from the rectum and the ureter. However, more extensive interventions with partial resection of the bowel or bladder can also be performed with lower complication rates by this minimally invasive approach. Furthermore, the excellent view and the precise navigation of instruments permit preservation of the ovarian reserve, optimal restoration of anatomical conditions, and the prevention of postoperative adhesions (13). Recent investigations have shown that robotic surgery achieves these goals in the vast majority of cases, such as the fulfillment of a woman's wish for children, a significant reduction of pain, and the improvement of gastrointestinal symptoms. Further studies in large cohorts of patients will show whether, and to what extent, robotic surgery is superior to a traditional laparoscopic procedure (14).

Urogynecological interventions can be performed safely, precisely, and rapidly with the robot. The results of sacrocolpopexy are similar to those obtained by open surgery. The robot is especially useful for better visualization and handling in the presacral region, as well as in the insertion of a mesh and its secure fixation to the vaginal stump, the cervical stump, or the presacral area (15).

Re-anastomosis of the fallopian tubes for the purpose of refertilization is associated with a success rate of $67.6 \%$ in open surgery, and a 5.6\% incidence of ectopic pregnancies. (16). Compared to conventional laparoscopy, the operation robot permits more "delicate" handling and a better view of the anatomical/histological layers, which is required for precise suturing and exact restoration of anatomical conditions (17). The disadvantage compared to the open procedure is the longer operating time. However, the duration of the hospital stay and the period of convalescence are markedly reduced (18). Longterm investigations will show whether robot-assisted surgery provides similar success rates as the open procedure. Notably, recent investigations revealed a re-anastomosis rate higher than $90 \%$ (19) (Figure 4, 5).

In obstetrics, cervical cerclage is another field of application for robotic surgery in order to avoid preterm births in patients with cervical insufficiency (20). The most common method is operation by vaginal access. When this is rendered impossible because of an excessively short cervix (caused by extensive conization, for instance) or for other technical reasons, abdominal access serves as an alternative (21). Here the advantages of the robotic procedure compared to the traditional laparoscopic approach include a much simpler intracorporeal suturing and more precise intraoperative exposure with less injury to the adjoining structures. A cerclage may be applied before, as well as during, a pregnancy. Successful pregnancies, near-term 

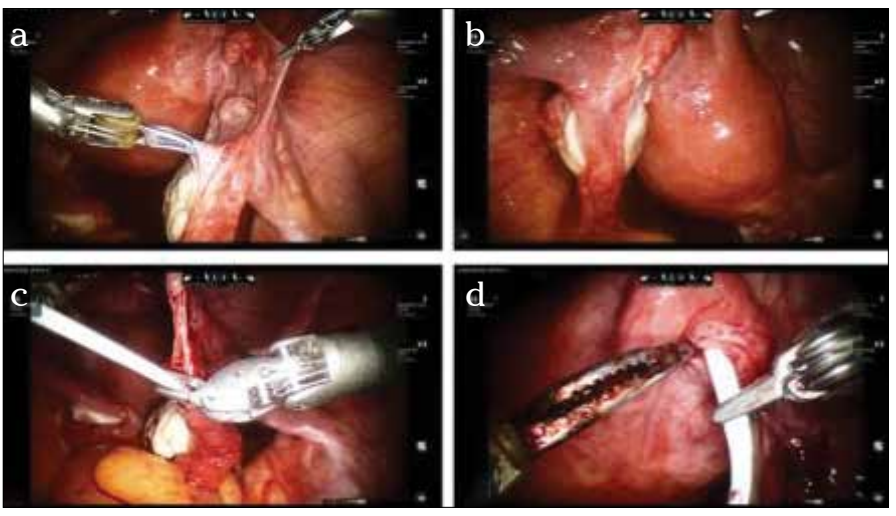

Figure 4. a-d. Operation site after laparoscopic sterilization on the right side (a) and the left side (b). After freshening the wound edges in the distal aspect and mobilization of the mesentery, a thin and blunt catheter is inserted into the distal end; the catheter protrudes from the fimbrial funnel of the fallopian tube (c). The other end of the catheter is pushed into the freshened proximal end so that it almost serves as a bridge (d).
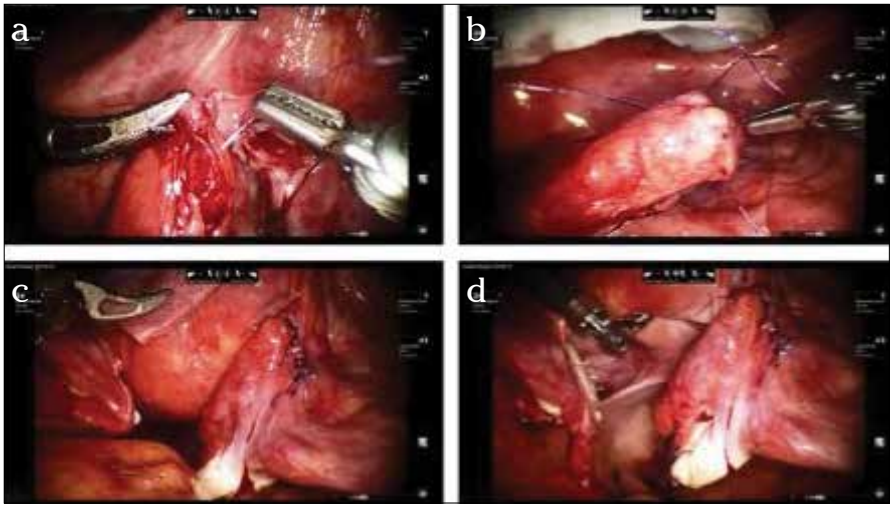

Figure 5. a-d. The first suture is made, strictly in the seromuscular aspect, omitting the mucosa. Very thin suture material or even monofilament sutures (at least 4-0) are suitable for this purpose (a). Four to eight sutures (b) that may then be knotted consecutively (c). Final situs on the left side after complete re-anastomosis (d). Bland suture and successful chromopertubation; no contrast medium emerges from the suture area and contrast medium is drained promptly through the fimbriae.

births, and low rates of preterm births have been reported for both procedures $(22,23)$. As the removal of the cerclage at the end of pregnancy is rendered very difficult by its intraabdominal positon, in these cases, a primary cesarean section is usually the only possible mode of delivery. Basically, the procedure is still in the experimental stage and should therefore be confined to individual cases after an exact estimation of the risks involved. It should be performed at centers specialized in the technique and in the course of clinical studies.

\section{Application in malignant gynecological disease}

The use of robotic surgery for malignant gynecological diseases is increasing in proportion to centralization and the availability of the robot. In the meantime, several retrospec- tive and comparative studies have shown that the oncological outcome of minimally invasive surgical procedures is at least equivalent to that of open surgery. This is true of the general advantages of minimally invasive surgery as well as specific oncological aspects, although long-term studies on this subject are largely unavailable yet. A few investigations have shown that, for instance, robotic radical hysterectomies appear to be equivalent to surgery by the abdominal approach in terms of long-term survival. In fact, the robotic procedure is possibly superior to the conventional procedure in terms of the number of resected lymph nodes $(7,24)$.

Despite the success of screening programs and HPV vaccination, cervical carcinoma continues to be a global problem. The feasibility of radical hysterectomy with the aid of the robot has been confirmed on several occasions. Compared to abdominal and conventional laparoscopic radical hysterectomy, the authors observed lesser blood loss, shorter operating times, lower complication rates, and shorter hospital stays (25). Nevertheless, the existing body of data is still heterogeneous. Besides, large randomized controlled studies that would eliminate bias on the part of individual surgeons and permit generalizations are still lacking (26).

The use of the robot may be meaningful even in fertility-conserving operations like trachelectomy. However, the majority of these operations are performed at individual centers. The three-dimensional image, large magnification, and tremor-free work ensure optimal visualization of vascular structures and the parametria, which must be partially preserved during the operation. The largest study published so far, based on two centers in Sweden, showed a pregnancy rate of $81 \%$ and a low rate of preterm births (71\% delivered their children after the $36^{\text {th }}$ gestational week) in 56 women (27).

The technique of total mesometrial resection (TMMR) of the uterus was first described by Michael Höckel and is now an established alternative in robot-assisted surgery for the treatment of cervical cancer. In contrast to the Wertheim-Meigs operation, it is not oriented to traditional anatomical margins, but integrates the principles of embryonic development into the concept of the morphogenetic uterovaginal unit. The data reported thus far indicate that the new therapy approach is associated with a large recurrence-free interval, better five-year survival rates, and lower morbidity rates compared to the traditional approach. These advantages are largely attributable to the absence of locoregional recurrence and the avoidance of additional adjuvant radiotherapy (28). TMMR can now also be performed by a minimally invasive approach (29).

Endometrial carcinoma is the most common malignant genital tumor among women in the Western world. At the time of diagnosis, the tumor is limited to the uterus in about $70 \%$ of cases. The primary therapy approach is surgery, consisting of hysterectomy with bilateral adenectomy. Surgery in endometrial cancer can be performed by the open approach, the minimally invasive approach (laparoscopic or robotic) or, in early tumor stage cases, even by the vaginal approach. Robotic hysterectomy with bilateral adenectomy was evaluated in a Danish study in regards to its impact on quality of life. A significant limitation of quality of life was noted one week postoperatively (56\% of the 


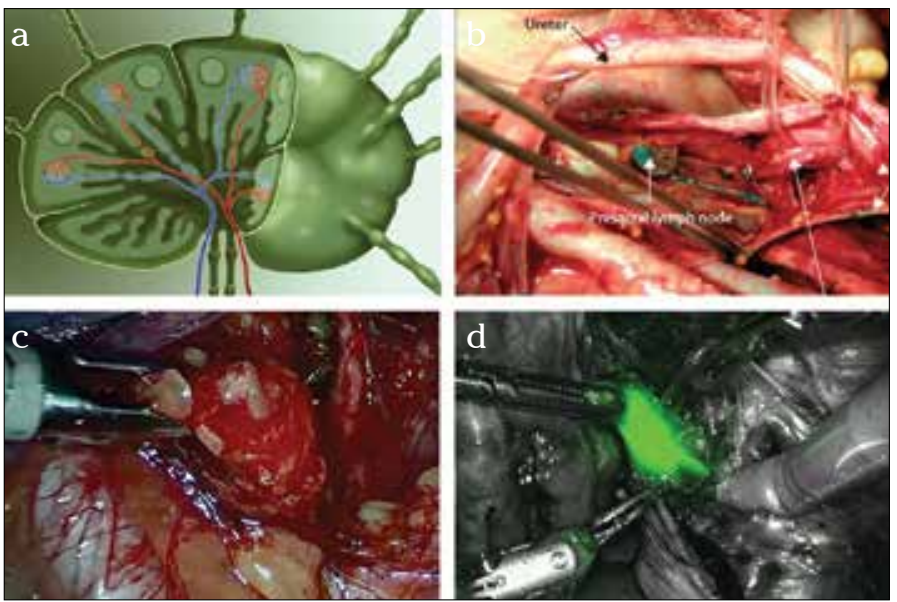

Figure 6. a-d. Schematic diagram of a lymph node. Several afferent lymph vessels from the peripheral aspect, central blood supply, and one or two efferent lymphatic vessels (a). Sentinel lymph nodes in endometrial cancer, here at the level of the common iliac artery on the right side, color-marked with methylene blue (b). In comparison: sentinel lymph node intraoperatively in endometrial cancer (c), partly marked with the fluorescent dye indocyanine green (d), here at the level of bifurcation of the common iliac artery on the right side.

baseline value). However, just 5 weeks post-surgery, the mean activity level of the patients were as high as $84 \%$, thus reaching the preoperative baseline value (30). Especially the depth of tumor infiltration and histological grading determine the need for simultaneous or two-stage pelvic and paraaortic lymphadenectomy. Robotic surgery achieved equivalent or better oncological results compared to laparoscopy. A Finnish investigation of 99 patients revealed no difference in the number of resected lymph nodes; but robotic surgery was associated with shorter operating times, fewer conversions to laparotomy, as well as fewer minor and major complications (31). In an American study, the robotassisted approach was found to be superior to the laparoscopic approach (32). The increasing use of minimally invasive surgical procedures also permits the application of sentinel node concepts. Sentinel nodes can be marked in color or by a fluorescent dye with the robot, and signify a further advancement compared to traditional laparoscopy (Figure 6) (33). Indocyanine green can also be used for intraoperative navigation, during ontogenetic compartment resection, in order to visualize the compartmental lymphatic system (34). Based on ontogenetic development, a new surgical concept has now been developed for compartment-based radical peritoneal mesometrial resection (PMMR) and therapeutic lymphadenectomy, according to Höckel, for the purpose of reducing locoregional recurrence. The efficacy of mesometrial resection of endometrial cancer is a part of ongoing studies in open as well as robotic surgery (35).

A large number of patients with endometrial cancer are overweight and obese. About $40 \%$ of the patients are overweight. The effort involved in the treatment of obese individuals is significantly greater than that for the treatment of non-obese patients with the same disease. Approximately one half of all endometrial cancers are operated upon with the minimally invasive approach. The limits of conventional laparoscopy become especially evident here. The general view of the lesser pelvis and the limited mobility of instruments have been reported as the primary limitations. These patients could especially benefit from the advantages of robotic surgery. Low complication rates have already been reported in many cases (36).

Other meaningful applications include anterior/posterior exenteration as interdisciplinary interventions. First described by Brunschwig in 1949, exenteration is still the treatment of choice for advanced or recurrent malignancies in the central compartment. Exenteration is frequently the sole potentially curative surgical method for recurrent cervical or endometrial cancer. Especially because of the ubiquitous use of laparoscopy in advanced urological disease and diseases calling for visceral surgery, the interdisciplinary approach permits the clinician to utilize the advantages of minimally invasive surgery (37).

The use of robotic surgery for the treatment of ovarian cancer is a debated issue, but should be viewed in the same context as the laparoscopic operation. At least for staging early carcinomas, as well as completion surgery for borderline tumors, the advantages of the minimally invasive approach are obvious. Simultaneously, there appears to be no evidence of any drawbacks. Attempts have also been made to use the robot for de-bulking surgery in ovarian cancer $(38,39)$. The necessity to operate in all four quadrants implies re-docking and rotation of the operating table. Since the body of data on this subject is still rather scarce, the patients should be selected individually; the procedure should undoubtedly be regarded as experimental (40).

Table 4 provides an overview of recent reports on the use of robotic surgery for malignant gynecological diseases.

\section{Application in complex situations}

In an age of increasing medical specialization and professionalization, the interdisciplinary approach is gaining importance. Even in surgical specialties, the use of a multimodality therapy concept and good cooperation between the individual specialties are becoming increasingly important (41). Especially, these facts offer new opportunities for robotic surgery. The direct division of the operation between surgeons of various specialties is rendered possible by two operation consoles that can be used simultaneously. This permits the specialists, who have different points of focus, to complement each other in symbiotic fashion. Furthermore, operating far from the field of operation enables a more rapid exchange of surgeons. Modern communication systems and telemonitoring have made it possible to exchange information over a large distance (42). In addition to saving resources and reducing costs, it is possible to work more effectively and provide better patient care.

Overweight patients constitute one of the greatest problems in health care and health care costs in the modern Western world. In Germany, approximately every second woman is overweight (BMI>25) and every fourth woman is obese (BMI>30) (43). In addition to the generally high health risk and resulting diseases, such as arterial hypertension, arteriosclerosis, and type II diabetes mellitus, obesity is a challenge in surgical specialties as well. On the one hand, overweight patients benefit from a minimally invasive approach in terms of morbidity and hospitalization. On 
Table 4. Selected studies on the use of robotic surgery in malignant gynecological disease

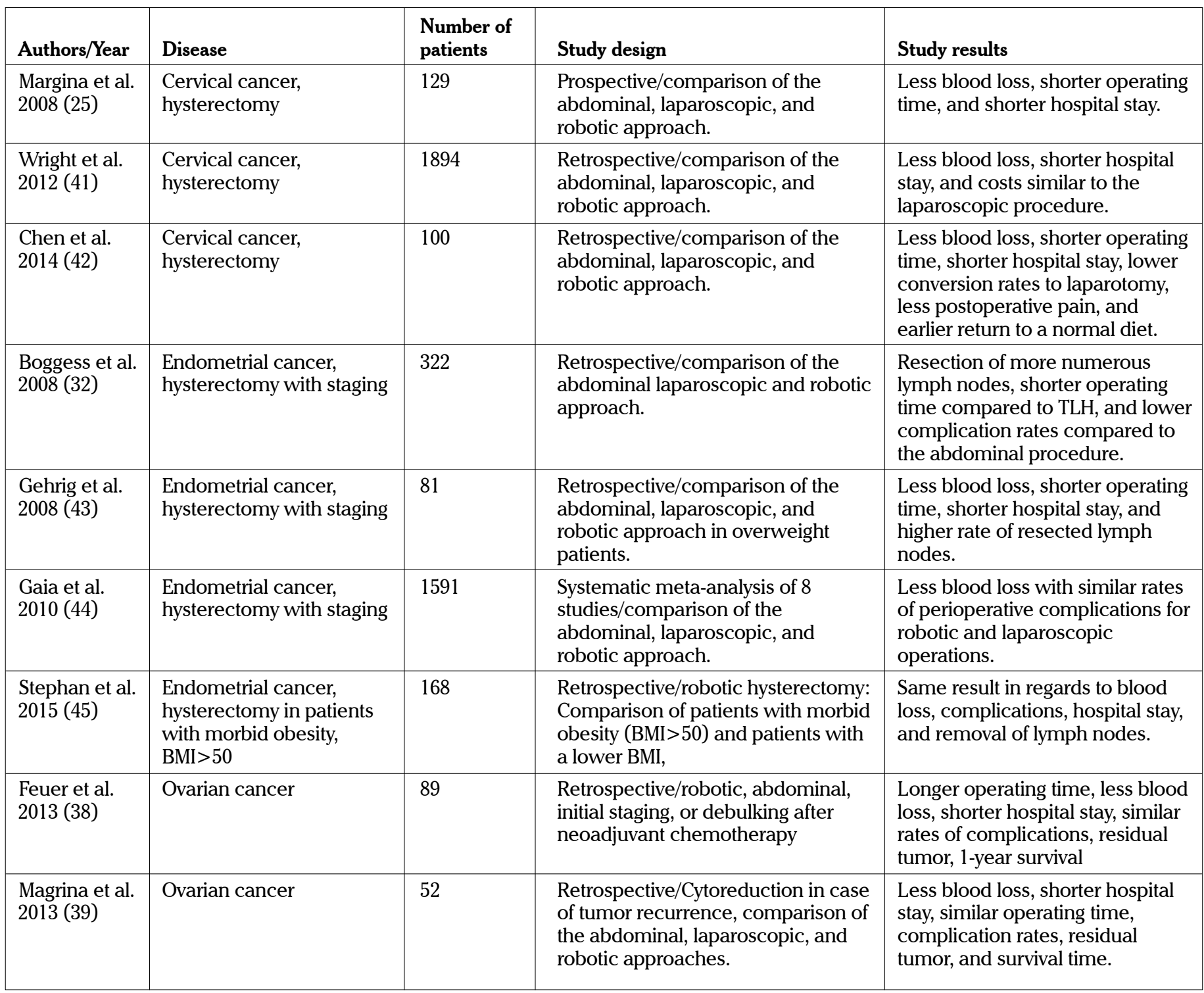

the other hand, these patients impose high demands on the surgeon and the operation system. Traditional laparoscopy reaches its limits here (4). Thanks to the robust surgical tools and 7 degrees of freedom (which dispense with the need for using the abdominal wall as a counter bearing) in robotic surgery, it can also be used in severely obese patients. The limitations imposed on the anesthetist by the extremely low head-down position have been largely overcome. Furthermore, shorter operating times are now possible with a comparable number of complications and hospital stays of similar duration $(44,45)$.

\section{Possibilities to improve surgical training in the age of minimally invasive surgery}

In order to obtain a good result with the Da Vinci operation robot, the surgeon first requires targeted training and sufficient initial practice. Knowledge of open surgery, vaginal surgery, and conventional laparoscopy are, of course, extremely important. Working at the operation console, at a distance from the patient, and the limited tactile feedback call for some readjustment on the part of the surgeon. However, the merits of the system create new opportunities in training and advanced training at all levels. The paradigm shift in the last few years has caused a growing generation of surgeons to primarily use laparoscopy instead of traditional open surgery. Classical training of anatomy-based fundamental surgical techniques and the positioning of the teacher's hand next to the student's hand are hindered to an increasing degree by mechanization. Thus, a basic understanding of anatomy and the specific properties of tissue, resulting from palpatory perception, is lacking in many cases and must be developed gradually. Hence, all surgical approaches retain their value. However, the basic mechanical skills of laparoscopy and robotic surgery can be acquired by introducing the surgeon early to virtual training programs and 
Table 5. Selected studies on the cost efficiency of robotic surgery in gynecology

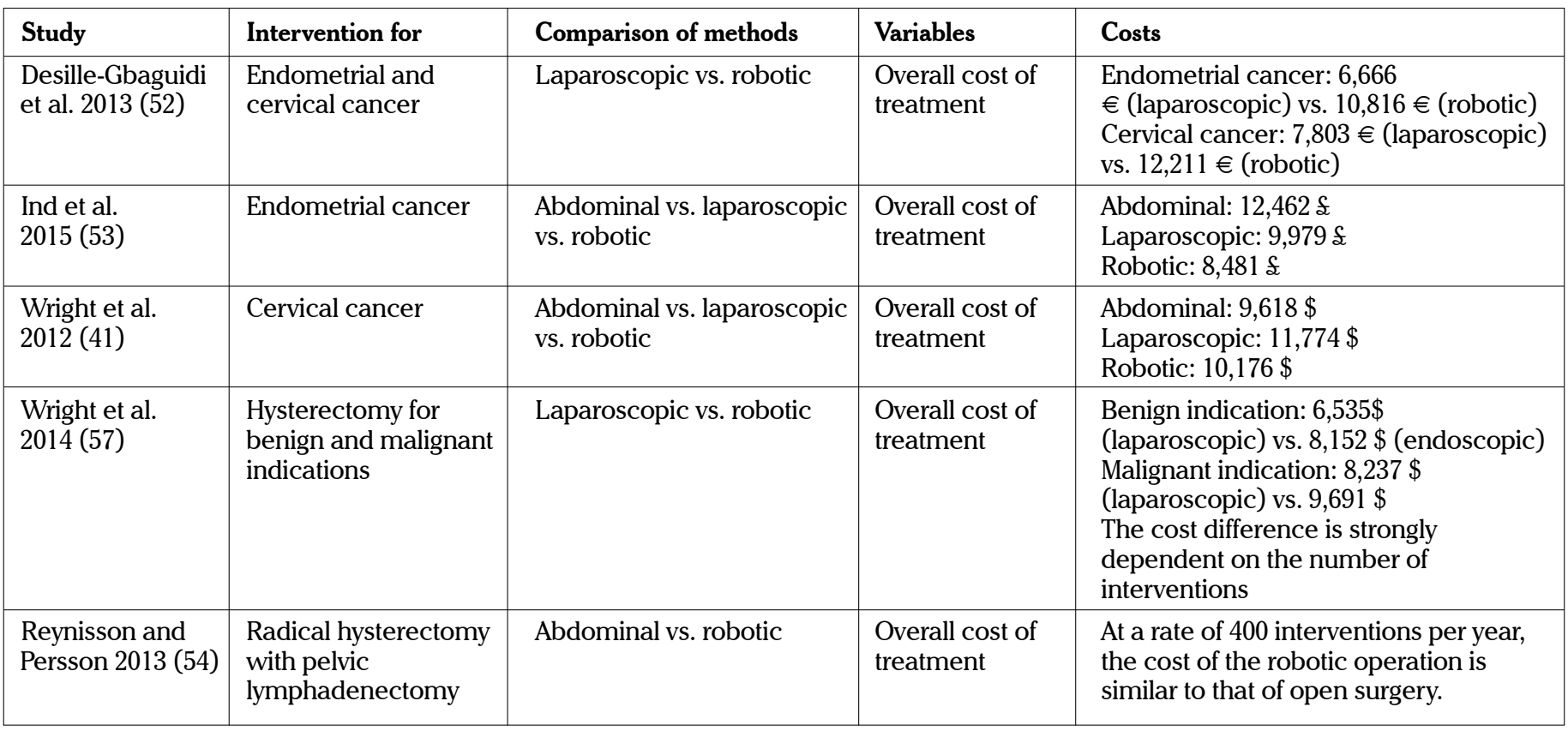

the pelvitrainer. Furthermore, the excellent imaging quality provides a clear view and enables the surgeon to learn about the relevant functional anatomy from a new perspective. The dual console of the robot signifies a quantum leap in practical surgical training. Two surgeons can work simultaneously, similar to the "driving school concept," and perform an operation together. Telemedicine will permit training and advanced training independent of space and time in the future (46).

\section{Cost efficiency of robotic surgery}

The most expensive aspect of robotic surgery, compared to all other surgical procedures, is the high cost of the equipment and the annual fixed costs (running costs and materials). In a study conducted by Desille-Gbaguidi, the cost of operations with the robot for endometrial cancer was 2.7-fold higher than that of conventional laparoscopy, and the cost of operations for cervical cancer was 2.6-fold higher than that of conventional laparoscopy. The overall cost of patient care was 1.6-fold higher (47). However, these calculations include the high acquisition cost of the equipment and, especially, the prolonged operating times. Furthermore, a variety of cost factors were included in the calculations. A careful analysis of endometrial cancer from Great Britain, however, has recently reported the contrary (48). There is consensus about the fact that maximum utilization of the system and a well-coordinated team with efficient processes of selection and organization can reduce the costs to the level of open surgical techniques. This is primarily achieved by progressively shorter operating times, fewer complications, and markedly shorter hospital stays. In the long term, more rapid convalescence and an earlier return to work also play a decisive role for the health care system (49). Attempts made thus far to compare conventional laparoscopy with robotic surgery in regards to costs have yielded very diverse results.
The use of disposable laparoscopic instruments, for instance, was not taken into account (50). Statistical projections about the required number of robotic interventions per year in order to achieve profitability range between 165 and 400 operations $(49,51)$. Critical studies on the cost efficiency of robotic surgery are shown in Table 5.

\section{Future perspectives}

The entry of alternative manufacturers in the field of robotic surgery will transform the cost situation in a groundbreaking manner. Increasing specialization on the one hand, and centralization on the other, will advance technological progress and digital networking of innovative surgical procedures more rapidly. Preliminary results obtained with the new Xi system have shown that the new system has been adopted rapidly and without difficulties in gyneco-oncology as well as in the interdisciplinary sector. Its high flexibility and satisfactory complication rates will lead to a larger volume of data in terms of results in the near future, which must then be sustained from the oncological point of view as well.

The fact that robot-assisted surgery complements, but does not replace, conventional laparoscopy is an undisputed fact.

Ethics Committee Approval: Ethics committee approval was received for this study from the ethics committee of the Christian Albrechts University in Kiel.

Informed Consent: Written informed consent was obtained from all patients who participated in this study.

Peer-review: Externally peer-reviewed.

Author Contributions: Concept - I.A., L.M.; Design - J.A., I.A.; Supervision - L.M., N.M.; Resources - I.A., N.M.; Materials - J.A., L.M., N.M., I.A.; Data Collection and/or Processing - J.A., I.A.; Analysis and/or Interpretation 
- J.A., L.M., N.M., I.A.; Literature Search - J.A., I.A.; Writing Manuscript J.A., Critical Review - L.M., N.M.

Acknowledgements: The authors would like to thank Dawn Rüther for editing the manuscript.

Conflict of Interest: No conflict of interest was declared by the authors.

Financial Disclosure: The authors declared that this study has received no financial support.

\section{References}

1. Liu H, Lu D, Wang L, Shi G, Song H, Clarke J. Robotic surgery for benign gynaecological disease. Cochrane Database Syst Rev 2012; 2: CD008978.

2. Schollmeyer T, Mettler L, Alkatout I. Roboterchirurgie in der Gynäkologie - Der Operateur am Schreibtisch. Der Gynäkologe 2011: 196-201.

3. Yuh B, Yu X, Raytis J, Lew M, Fong Y, Lau C. Use of a mobile towerbased robot--The initial Xi robot experience in surgical oncology. $\mathrm{J}$ Surg Oncol 2016; 113: 5-7.

4. Sinha R, Sanjay M, Rupa B, Kumari S. Robotic surgery in gynecology. J Minim Access Surg 2015; 11: 50-9.

5. Herron DM, Marohn M; SAGES-MIRA Robotic Surgery Consensus Group. A consensus document on robotic surgery. Surg Endosc 2008; 22: 313-25; discussion 311-2.

6. Soliman PT, Langley G, Munsell MF, Vaniya HA, Frumovitz M, Ramirez PT. Analgesic and antiemetic requirements after minimally invasive surgery for early cervical cancer: a comparison between laparoscopy and robotic surgery. Ann Surg Oncol 2013; 20: 1355-9.

7. Sinno AK, Fader AN. Robotic-assisted surgery in gynecologic oncology. Fertil Steril 2014; 102: 922-32.

8. Venkat P, Chen LM, Young-Lin N, Kiet TK, Young G, Amatori D, et al. An economic analysis of robotic versus laparoscopic surgery for endometrial cancer: costs, charges and reimbursements to hospitals and professionals. Gynecol Oncol 2012; 125: 237-40.

9. Nezhat C, Saberi NS, Shahmohamady B, Nezhat F. Robotic-assisted laparoscopy in gynecological surgery. JSLS 2006; 10: 317-20.

10. Lim PC, Crane JT, English EJ, Farnam RW, Garza DM, Winter ML, et al. Multicenter analysis comparing robotic, open, laparoscopic, and vaginal hysterectomies performed by high-volume surgeons for benign indications. Int J Gynaecol Obstet 2016; 133: 359-64.

11. Soto E, Flyckt R, Falcone T. Endoscopic management of uterine fibroids: an update. Minerva Ginecol 2012; 64: 507-20.

12. Pitter MC, Gargiulo AR, Bonaventura LM, Lehman JS, Srouji SS. Pregnancy outcomes following robot-assisted myomectomy. Hum Reprod 2013; 28: 99-108.

13. Siesto G, Ieda N, Rosati R, Vitobello D. Robotic surgery for deep endometriosis: a paradigm shift. Int J Med Robot 2014; 10: p. 140-6.

14. Abo C, Roman H, Bridoux V, Huet E, Tuech JJ, Resch B, et al. Management of deep infiltrating endometriosis by laparoscopic route with robotic assistance: 3-year experience. J Gynecol Obstet Biol Reprod (Paris) 2016; pii: S0368-2315(15)00380-4.

15. Ayav A, Bresler L, Hubert J, Brunaud L, Boissel P. Robotic-assisted pelvic organ prolapse surgery. Surg Endosc 2005; 19: 1200-3.

16. Abelha Mde C, Costa RR, Lopes VM, Reis RC, Silva CM. [Tubal reanastomosis: analysis of the results of 30 years of treatment]. Rev Bras Ginecol Obstet 2008; 30: 294-9.

17. Rodgers AK, Goldberg JM, Hammel JP, Falcone T. Tubal anastomosis by robotic compared with outpatient minilaparotomy. Obstet Gynecol 2007; 109: 1375-80.

18. Bedaiwy MA, Barakat EM, Falcone T. Robotic tubal anastomosis: technical aspects. JSLS 2011; 15: 10-5.
19. Kavoussi SK, Kavoussi KM, Lebovic DI. Robotic-assisted tubal anastomosis with one-stitch technique. J Robot Surg 2014; 8: 133-6.

20. Moore ES, Foster TL, McHugh K, Addleman RN, Sumners JE. Robotic-assisted transabdominal cerclage (RoboTAC) in the non-pregnant patient. J Obstet Gynaecol 2012; 32: 643-7.

21. DaCosta V, Wynter S, Harriott J, Christie L, Frederick J, FrederickJohnston S. Laparoscopic cervicoisthmic cerclage for the treatment of cervical incompetence: case reports. West Indian Med J 2011; 60: 590-3.

22. Gocmen A, Sanlikan F. Two Live Births following Robotic-Assisted Abdominal Cerclage in Nonpregnant Women. Case Rep Obstet Gynecol 2013; 2013: 256972.

23. Walsh TM, Borahay MA, Fox KA, Kilic GS. Robotic-assisted, ultrasound-guided abdominal cerclage during pregnancy: overcoming minimally invasive surgery limitations? J Minim Invasive Gynecol 2013; 20: 398-400.

24. Brudie LA, Backes FJ, Ahmad S, Zhu X, Finkler NJ, Bigsby GE 4th, et al., Analysis of disease recurrence and survival for women with uterine malignancies undergoing robotic surgery. Gynecol Oncol 2013; 128: 309-15.

25. Magrina JF, Kho RM, Weaver AL, Montero RP, Magtibay PM. Robotic radical hysterectomy: comparison with laparoscopy and laparotomy. Gynecol Oncol 2008; 109: 86-91.

26. Magrina, JF, Zanagnolo VL. Robotic surgery for cervical cancer. Yonsei Med J 2008; 49: 879-85.

27. Johansen G1, Lönnerfors C2, Falconer H1, Persson J. Reproductive and oncologic outcome following robot-assisted laparoscopic radical trachelectomy for early stage cervical cancer. Gynecol Oncol 2016; 141: 160-5.

28. Höckel M, Horn LC, Manthey N, Braumann UD, Wolf U, Teichmann $\mathrm{G}$, et al. Resection of the embryologically defined uterovaginal (Mullerian) compartment and pelvic control in patients with cervical cancer: a prospective analysis. Lancet Oncol 2009; 10: 683-92.

29. Kimmig R, Wimberger P, Buderath P, Aktas B, Iannaccone A, Heubner M. Definition of compartment-based radical surgery in uterine cancer: radical hysterectomy in cervical cancer as 'total mesometrial resection (TMMR)' by M Hockel translated to robotic surgery (rTMMR). World J Surg Oncol 2013; 11: 211.

30. Herling SF, Møller AM, Palle C, Thomsen T. Health-related quality of life after robotic-assisted laparoscopic hysterectomy for women with endometrial cancer--A prospective cohort study. Gynecol Oncol 2016; 140: 107-13.

31. Mäenpää MM, Nieminen $K$, Tomás EI, Laurila $M$, Luukkaala $T H$, Mäenpää JU. Robotic-assisted vs traditional laparoscopic surgery for endometrial cancer: a randomized controlled trial. Am J Obstet Gynecol 2016; 215: 588.e1-588.e7.

32. Boggess JF, Gehrig PA, Cantrell L, Shafer A, Ridgway M, Skinner EN, et al. A comparative study of 3 surgical methods for hysterectomy with staging for endometrial cancer: robotic assistance, laparoscopy, laparotomy. Am J Obstet Gynecol 2008; 199: 360.e1-9.

33. Morice P, Leary A, Creutzberg C, Abu-Rustum N, Darai E. Endometrial cancer. Lancet 2016; 387: 1094-108.

34. Kimmig R, Aktas B, Buderath P, Rusch P, Heubner M. Intraoperative navigation in robotically assisted compartmental surgery of uterine cancer by visualisation of embryologically derived lymphatic networks with indocyanine-green (ICG). J Surg Oncol 2016; 113: 554-9.

35. Kimmig R, Iannaccone A, Aktas B, Buderath P, Heubner M. Embryologically based radical hysterectomy as peritoneal mesometrial resection (PMMR) with pelvic and para-aortic lymphadenectomy for loco-regional tumor control in endometrial cancer: first evidence for efficacy. Arch Gynecol Obstet 2016; 294: 153-60.

36. Chan JK, Gardner AB, Taylor K, Thompson CA, Blansit K, Yu X, et al. Robotic versus laparoscopic versus open surgery in morbidly 
obese endometrial cancer patients - a comparative analysis of total charges and complication rates. Gynecol Oncol 2015; 139: 300-5.

37. Schneider A, Köhler C, Erdemoglu E. Current developments for pelvic exenteration in gynecologic oncology. Curr Opin Obstet Gynecol 2009; 21: 4-9.

38. Feuer GA, Lakhi N, Barker J, Salmieri S, Burrell M. Perioperative and clinical outcomes in the management of epithelial ovarian cancer using a robotic or abdominal approach. Gynecol Oncol 2013; 131: 520-4.

39. Magrina JF, Cetta RL, Chang YH, Guevara G, Magtibay PM. Analysis of secondary cytoreduction for recurrent ovarian cancer by robotics, laparoscopy and laparotomy. Gynecol Oncol 2013; 129: 336-40.

40. Chen CH1, Chiu LH, Chen HH, Chan C1, Liu WM. Comparison of robotic approach, laparoscopic approach and laparotomy in treating epithelial ovarian cancer. Int J Med Robot 2016; 12: 268-75.

41. Alkatout I, Egberts JH, Mettler L, Doniec M, Wedel T, Jünemann KP, et al. [Interdisciplinary Diagnosis and Treatment of Deep Infiltrating Endometriosis.]. Zentralbl Chir 2015.

42. Sebajang H, Trudeau P, Dougall A, Hegge S, McKinley C, Anvari M. The role of telementoring and telerobotic assistance in the provision of laparoscopic colorectal surgery in rural areas. Surg Endosc 2006; 20: 1389-93.

43. Institut, R.K. Übergewicht und Adipositas. 2016 21.01.2016]; Available from: http://www.rki.de/DE/Content/Gesundheitsmonitoring/Themen/ Uebergewicht_Adipositas/Uebergewicht_Adipositas_node.html.
44. Scheib SA, Tanner E 3rd, Green IC, Fader AN. Laparoscopy in the morbidly obese: physiologic considerations and surgical techniques to optimize success. J Minim Invasive Gynecol 2014; 21: 182-95.

45. Siesto G, Ornaghi S, ledà N, Vitobello D. Robotic surgical staging for endometrial and cervical cancers in medically ill patients. Gynecol Oncol 2013; 129: 593-7.

46. Goonewardene SS, Brown M, Challacombe B. Challacombe, Single- versus dual-console robotic surgery: dual improves the educational experience for trainees. World J Urol 2016; 34: 1337-9.

47. Desille-Gbaguidi H, Hebert T, Paternotte-Villemagne J, Gaborit C, Rush E, Body G. Overall care cost comparison between robotic and laparoscopic surgery for endometrial and cervical cancer. Eur J Obstet Gynecol Reprod Biol 2013; 171: 348-52.

48. Ind TE, Marshall C, Hacking M, Harris M, Bishop L, Barton D, et al. Introducing robotic surgery into an endometrial cancer service-a prospective evaluation of clinical and economic outcomes in a UK institution. Int J Med Robot 2016; 12: 137-44.

49. Reynisson P, Persson J. Hospital costs for robot-assisted laparoscopic radical hysterectomy and pelvic lymphadenectomy. Gynecol Oncol 2013; 130: 95-9.

50. Alkatout I, Mettler L, Günther V, Maass N, Eckmann-Scholz C, Elessawy $\mathrm{M}$, et al. Safety and economical innovations regarding surgical treatment of fibroids. Minim Invasive Ther Allied Technol 2016; 25: 1-13.

51. Marino P, Houvenaeghel G, Narducci F, Boyer-Chammard A, Ferron G, Uzan C, et al. Cost-Effectiveness of Conventional vs RoboticAssisted Laparoscopy in Gynecologic Oncologic Indications. Int J Gynecol Cancer 2015; 25: 1102-8. 